\title{
Effect of transient exposure to carbaryl wettable powder on the gut microbial community of honey bees
}

\author{
Kathyleen Nogrado ${ }^{1}$, Seul Lee ${ }^{1}$, Kyongmi Chon ${ }^{2^{*}}$ and Ji-Hoon Lee ${ }^{1^{*}}$ (D)
}

\begin{abstract}
Bees are important pollinators in agriculture. The bee population has recently begun to decline possibly due to pesticides. The bee gut microbiota strongly influences the health of bees. The gut microbiota of bees is composed of distinct members belonging to selective taxa. Chemicals like pesticides can alter the gut microbiota. The present study investigated the effect of carbaryl pesticides on gut microbiota of honey bees, which had come in contact with rapeseed plants (Brassica napus) sprayed with carbaryl wettable powder during the honey bee brood test under semifield condition. Molecular techniques (conventional and quantitative polymerase chain reaction (PCR), clone library method, and DNA sequencing) were employed to analyze changes in the microbial communities between the pesticide-exposed and unexposed bees. Phylogenetic analysis of 16S rRNA genes of the clones from both groups, showed differences in their respective compositions of core and non-core bacteria. Both groups contained carbohydratedegrading bacteria such as Gilliamella apicola and Lactobacillus. However, the unexposed bees harbored Alphaproteobacteria, which were absent in the exposed bees. Microorganisms found in honey bee guts such as Snodgrassella alvi and L. kullabergensis, however, were observed only in the exposed bees, but not in the unexposed bees. The difference between the two groups was distinctly recognized when copy numbers of 165 rRNA genes were compared by quantitative PCR. Results showed that the average gene copy number for the unexposed bees was higher than that for the exposed bees. This may indicate the toxic effect of pesticides on bees and gut microbiota.
\end{abstract}

Keywords: Gut microbiota, Honey bee, Pesticide, Toxicity

\section{Introduction}

Pollinators that move pollen from anther to stigma in flowers include ants, bats, beetles, birds, butterflies, flies, moths, wasps, and bees. Among these organisms, bees play a major role as pollinators in the agricultural sector [1-3]. One-third of the crops for food consumption rely on bee pollination. A rough estimation of their contribution to the economy worldwide is more than $\$ 200$ billion $[4,5]$. In recent years, there has been a decline

\footnotetext{
*Correspondence: kmchon6939@korea.kr; jhlee2@jbnu.ac.kr

1 Department of Bioenvironmental Chemistry, Chonbuk National

University, 567 Baekje-daero, Deokjin-gu, Jeonju-si, Jeollabuk-do 54896, Republic of Korea

${ }^{2}$ Chemical Safety Division, Department of Agro-Food Safety and Crop Protection, National Institute of Agricultural Sciences, Rural Development Administration, 166, Nongsaengmyeong-ro, Iseo-myeon, Wanju-gun, Jeollabuk-do 55365, Republic of Korea
}

in the population of both feral and managed bees [1, 2, 5]. Diverse causes have been proposed for the decline, including pests such as Varroa mites, diseases such as the foul brood, and pesticides [6-8]. Use of pesticides for crop protection has affected non-target organisms such as bees. Exposure of bees to pesticides would involve direct acute exposure as well as steady accumulation of the agrochemicals [9]. An example of this agrochemical is carbamates which have been commonly applied as insecticides among orchards. However, timing of the carbamates application should be taken into consideration especially during the stage of blossom [10]. The members of the honey bee hives subjected to high incidence of exposure to pesticides are the worker bees $[2,11]$. This could be supported by the study of Johnson et al. [2] stating that carbaryl was detected up to about $1.4 \mathrm{ppm}$ from 
pollen in hives. The worker bees collect nectar and pollen from different flowers and bring it back to the colony hives. They are also tasked with looking after the larvae, specifically in feeding them through the process known as trophallaxis [12]. This feeding behavior strongly influences the development of microbial community in guts of the juvenile honey bees. The uniformity of microbial composition in the guts of Apis and non-Apis bees is a result of this feeding behavior $[13,14]$.

The significance of gut microbiota lies in its symbiotic relationship with the host, and one familiar example for this is the beneficial flora in human gut $[15,16]$. Likewise, in lower organism, the host insect provides the microbiota with the necessary nutrients. In return, the microorganisms metabolize the substances that the hosts are usually incapable of digesting [17]. Gut microbiota also provides protection from the invasion of opportunistic pathogenic bacteria, which the insects acquire from the environment [12]. These symbiotic microbes, initially established in the gut, prevent the proliferation of unwanted microorganisms [18]. Similar to other organisms, the health of the honey bees is greatly influenced by the gut microbiota. Disruption of balance in gut microbiome or dysbiosis could result in accrued susceptibility of honey bees to diseases and pathogenic microorganisms $[12,13,19]$. The gut microbiota of honey bees is less complex but highly specialized compared to gut microbiota of other organisms $[3,15]$. It has been reported that the honey bee gut microbiota is dominated by nine bacterial species clusters, each species performing specific functions relating to carbohydrate breakdown, host defense, and immunity $[3,17,18]$.

Recent studies have given significant attention to the identification of the factors affecting the lability of the composition of the symbiotic gut microbes. One such factor is exposure to chemicals such as pesticides and antibiotics $[13,19]$. This exposure includes not only the direct application of the chemicals onto the hives but also the pesticides-applied plants, that the honey bees come in contact with during nectar collection [2, 17]. Exposure to these chemicals can possibly alter the gut microbial composition of honey bees, consequently affecting immune function, metabolism, and susceptibility of these bees to pathogens as well [12].

Since the composition of the gut microbiome of honey bees is rather simple compared to the other organisms, shifts in the microbial communities can be detected more easily. In the present study, the effect of carbaryl, a broad-spectrum insecticide, on the size and composition of bacterial community in the guts of honey bees was investigated.

\section{Materials and methods \\ Exposure of honey bees to pesticide under semi-field conditions}

The semi-field test was performed to assess the effect of carbaryl wettable powder (WP) 50\%, Detailed experimental conditions were maintained as described previously [4]. The experiment consisted of three treatment groups (negative control without pesticide exposure, positive control treated with diflubenzuron WP, and the experiment treated with carbaryl WP), with each group in three replicate tunnels. The honey bee colonies were placed in tunnels covering an area of $70 \mathrm{~m}^{2}$ and containing Brassica napus. Negative controls were sprayed with tap water $(400 \mathrm{~L} / \mathrm{ha})$, while the experiments were sprayed with carbaryl ( $250 \mathrm{~g}$ a.i./ha in $400 \mathrm{~L}$ tap water/ha) during active flight of bees. Bees were collected from the negative control and the carbaryl-treated groups, after $2 \mathrm{~h}$ of exposure.

\section{Genomic DNA extraction, PCR and clone library construction}

The collected bees were placed in $50-\mathrm{mL}$ conical centrifuge tubes and kept in $-80{ }^{\circ} \mathrm{C}$ prior to dissection. Each honey bee was surface sterilized by washing with $70 \%$ ethanol and rinsed with sterile distilled water. A total of 10 honey bees from each group were dissected individually in $70 \%$ ethanol to isolate the gut. All the isolated guts were pooled in a $1.5-\mathrm{mL}$ microcentrifuge tube. Total genomic DNA from the pooled isolated guts was extracted according to the manufacturer's instructions indicated in PowerSoil ${ }^{\circledR}$ DNA Isolation Kit (MO BIO Laboratories, Inc., Carlsbad, CA, USA).

The 16S rRNA gene was amplified using the bacterial universal primers $27 \mathrm{~F}$ and $1492 \mathrm{R}$ in $\mathrm{T} 100^{\mathrm{TM}}$ Thermal Cycler (Bio-Rad Laboratories, Inc., Forster City, CA, USA). The PCR conditions were as follows: initial denaturation at $95^{\circ} \mathrm{C}$ for $1 \mathrm{~min}$, followed by 30 cycles of denaturation at $95^{\circ} \mathrm{C}$ for $30 \mathrm{~s}$, annealing at $55^{\circ} \mathrm{C}$ for $30 \mathrm{~s}$, and elongation at $72{ }^{\circ} \mathrm{C}$ for $5 \mathrm{~min}$. The final extension was set at $72{ }^{\circ} \mathrm{C}$ for $5 \mathrm{~min}$.

Clone libraries were constructed for 16S rRNA genes from the two groups of genomic DNAs. PCR products of 16S rRNA gene were ligated into pLUG-Prime ${ }^{\circledR}$ TA cloning vector (iNtRON Biotechnology, Seongnam, South Korea). The ligated PCR products in the plasmid were then transformed into chemically competent Escherichia coli DH5- $\alpha$ cells (Enzynomics, Daejeon, South Korea). The transformed cells were plated onto Lysogeny (LB) agar, supplemented with $100 \mu \mathrm{g} / \mathrm{mL}$ ampicillin (iNtRON Biotechnology, Seongnam, South Korea). Clones containing the insert were selected by blue and white screening. 


\section{DNA sequencing and phylogenetic analysis}

In order to confirm the insert DNA, randomly selected representative white colonies were subjected to colony PCR using the primers M13F and M13R, and the fragment size of PCR products was checked using agarose gel electrophoresis. After confirmation of the desired band, the PCR products from all the selected white colonies were purified using AccuPrep ${ }^{\circledR}$ PCR Purification Kit (Bioneer Corporation, Daejeon, South Korea). Following quantification of DNA concentration using Qubit ${ }^{\circledR} 3.0$ Fluorometer (Thermo Fisher Scientific, Waltham, MA, US), the purified DNA samples were sent for sequencing at Genotech (Daejeon, South Korea).

The obtained contigs for the DNA sequences were first assembled using BioEdit version 7.0.5.3, followed by manual editing. The edited assembled DNA sequences were then searched for similar DNA sequences using National Center for Biotechnology Information (NCBI) BLAST. The sequences of both the clones and the obtained similar sequences were aligned using MUSCLE in MEGA7. The phylogenetic trees were then constructed using the neighbor-joining clustering method and Kimura 2-parameter distance model with 1000 bootstrap replications.

DNA sequences from the clones were deposited at NCBI GenBank with accession numbers MH842171 to MH842195 and MH879846 to MH879858.

\section{Quantitative real-time PCR}

For quantification of $16 \mathrm{~S}$ rRNA gene, quantitative realtime PCR (qPCR) was performed with a standard curve for absolute quantification in CFX Connect ${ }^{\mathrm{TM}}$ Real-Time PCR Detection System (Bio-Rad Laboratories, Inc., Forster City, CA, USA). The $16 \mathrm{~S}$ rRNA gene region was first amplified with some modification from genomic DNA of E. coli DH5- $\alpha$ using PCR with primers $27 \mathrm{~F}$ and $519 \mathrm{R}$ [20]. The 16S rRNA gene was cloned into the plasmid as described above and subsequently transformed into competent cells. The plasmid containing 16S rRNA gene (491 bp using 27F and 519R) was utilized to construct the standard curve for absolute quantification of $16 \mathrm{~S}$ rRNA gene copy numbers. Five series of 10 -fold dilution of concentration of the plasmid with the inserted $16 \mathrm{~S}$ rRNA gene, with three replicates, were analyzed. The master mix for the qPCR was prepared as follows: a total of $25 \mu \mathrm{L}$ consisting of $10 \mu \mathrm{L}$ AccuPower ${ }^{\circledR} 2 \mathrm{X}$ GreenStar qPCR Master Mix (Bioneer Corporation, Daejeon, South Korea), $1 \mu \mathrm{L}$ each of $27 \mathrm{~F}$ and $519 \mathrm{R}(10 \mu \mathrm{M}), 8 \mu \mathrm{L}$ sterile distilled water, and $5 \mu \mathrm{L}$ plasmid template. The conditions for the qPCR were set as follows: initial denaturation at $95^{\circ} \mathrm{C}$ for $5 \mathrm{~min}, 24$ cycles of denaturation at $94{ }^{\circ} \mathrm{C}$ for $30 \mathrm{~s}$, annealing at $58^{\circ} \mathrm{C}$ for $40 \mathrm{~s}$, and extension at $72{ }^{\circ} \mathrm{C}$ for $1 \mathrm{~min}$. The melting curve analysis was set between 65 and $95{ }^{\circ} \mathrm{C}$ with an increment of $0.5^{\circ} \mathrm{C}$ for $5 \mathrm{~s}$. Gene copy number was calculated using the cycle of quantification (Cq) based on the formula indicated by Ritalahti et al. [21]. For the bee samples, qPCR was performed using the same conditions.

\section{Statistical analysis}

The single-factor analysis of variance (ANOVA) was performed for the statistical analysis of the gene copy number in Microsoft Excel.

\section{Results}

\section{Bacterial community composition}

Thirty clones from each clone library of the exposed and unexposed groups were used for the DNA sequence analysis. The primers $27 \mathrm{~F}$ and $1492 \mathrm{R}$ were used to amplify the entire hypervariable regions V1-V9 of the $16 \mathrm{~S}$ rRNA gene. Each of the 30 sequences from the unexposed and

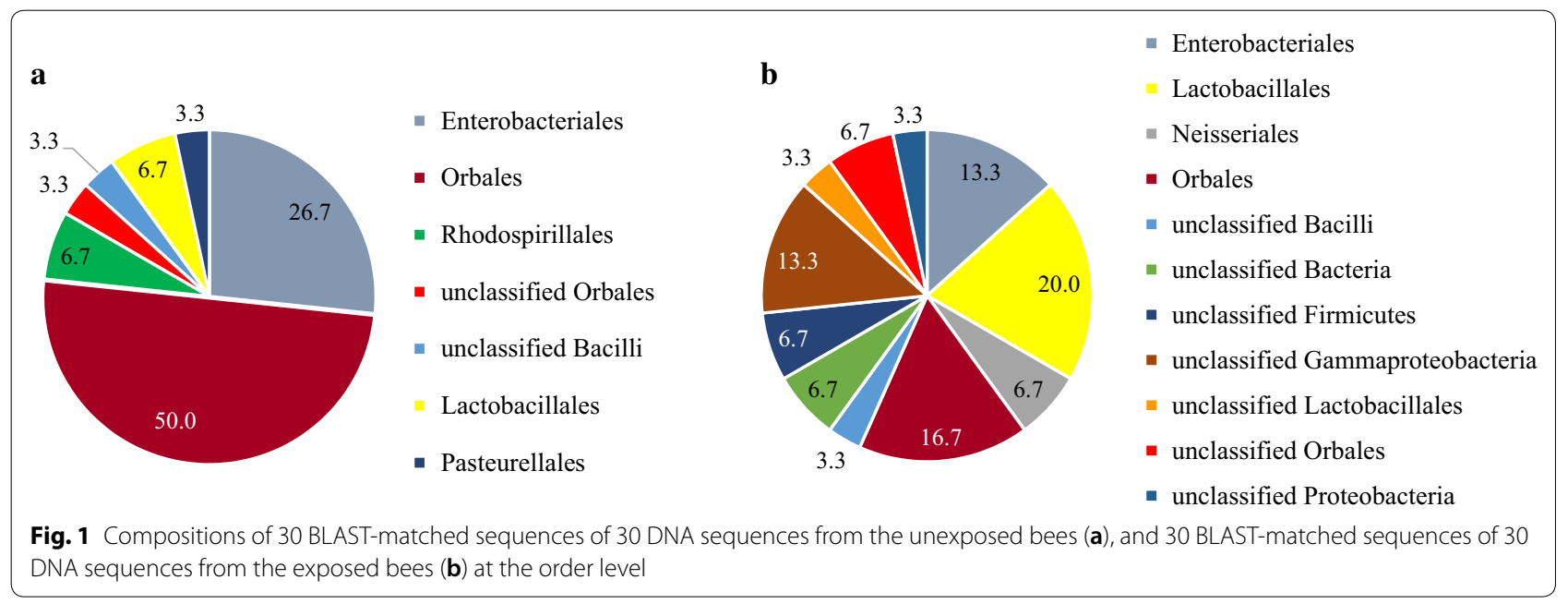


exposed bees was compared with the most similar DNA sequence retrieved from BLAST. Both groups showed bacteria belonging to Enterobacteriales, Lactobacillales, and Orbales as the common taxonomic groups. Moreover, the latter two taxa composed the majority of the identity of bacterial species in the unexposed and exposed bees. However, DNA sequences from the unexposed bees were more diverse at bacterial species than those observed in the exposed bees (Fig. 1). The taxonomic groups detected in the unexposed bees which consisted of Bacillales, Pasteurellales, and Rhodospirillales were absent in the exposed bees. Conversely, species from the order Neisseriales were detected only in the exposed bees, but not in the unexposed bees.

\section{Phylogenetic analysis of the clone sequences}

Differences in the composition of microbial communities between the bee groups were examined based on phylogenetic affiliations. The phylogenetic tree for the unexposed group was split into two as there were nine clones that diverged from the main tree. However, the second tree for the unexposed bees constructed for these nine clones still diverged from the second set of reference bacteria. Hence, this tree was not used for describing the microbial community in the unexposed bees. On the other hand, there was only one phylogenetic tree constructed for the exposed bees.

In the phylogenetic tree of the unexposed bees (Fig. 2), there were six clusters observed. These clusters were grouped into three classes. The first group was class Alphaproteobacteria with sequences similar to a genus Commensalibacter. The second cluster was class Bacilli including sequences similar to genus Lactobacillus. The remaining four clusters were grouped under class Gammaproteobacteria composed mainly of sequences similar to Gilliamella apicola, Frischella perrara, and Klebsiella oxytoca. There was only one phylogenetic tree generated for the exposed bee group (Fig. 3). In comparison to the phylogenetic tree of the unexposed bees, more clusters were observed in the exposed bees, but the nine clusters could further be grouped into three taxonomic classes; namely classes Bacilli, Gammaproteobacteria, and Betaproteobacteria. Interestingly, class Alphaproteobacteria was not observed in the exposed bees, and Betaproteobacteria was only recognized in the exposed bees. Although order Enterobacteriales was observed in both the unexposed and the exposed bees, more genera were distinguished in the exposed bees as exemplified by Cronobacter, Edwardsiella, Erwinia, Pantoea, Providencia, Serratia, and Snodgrasella. In addition, the copy number of the 16S rRNA gene per honey bee gut was analyzed in both groups to suggest relative bacterial abundance between the two groups. The average gene copy number of $16 \mathrm{~S}$ rRNA gene was higher in the unexposed bees $\left(2.83 \times 10^{6} \pm 1.20 \times 10^{6}\right.$ per gut $)$ as compared with that in the exposed bees $\left(5.25 \times 10^{5} \pm 9.81 \times 10^{3}\right.$ per gut) (Fig. 4). The single-factor ANOVA showed that the difference in the average gene copy of $16 \mathrm{~S}$ rRNA gene was statistically significant with a $p$ value of 0.028 at $95 \%$ confidence interval.

\section{Discussion}

Previous studies have shown detrimental effects of carbaryl to honey bees. In this study, the potential effect of exposure to carbaryl pesticide on the health condition of the honey bee was looked into. The compositions of microbial communities in both the unexposed and exposed groups of bees were examined by comparing phylogenetic trees from randomly selected clones. According to Engel and Moran [22], bacterial community of honey bee gut was suggested to be composed of only nine species. These species were mostly members of phyla Firmicutes and Bacteroidetes; classes Gammaproteobacteria, Betaproteobacteria, and Alphaproteobacteria; and genus Bifidobacterium [12, 23, 24]. In this study, these bacterial groups, except for Bifidobacterium and Bacteroidetes, were recognized in both the bee groups. The two bacterial groups in exception have been reported to be often less abundant in bee microbiota [3].

Recently, Bonilla-Rosso and Engel [24] further categorized them into core and non-core members of the gut microbiota. The core members included Gilliamella apicola, Lactobacillus Firm-5 and Firm-4, Snodgrassella alvi, and Bifidobacterium. These core microbial members facilitate the acquisition of nutrients via carbohydrate metabolism [17]. The results of this study showed slight differences with respect to the previously described core gut microbial taxa. In this study, G. apicola and Lactobacillus were observed in both the groups. This was in accordance with the results of previous studies, since the two bacterial taxa have been known to be associated with the metabolism of sugars, derived from nectar, honey, or pollen present in honey bee diets [12, 17, 24]. Additionally, G. apicola is also known to contain pectinase, which catabolizes pectin, a component of pollen. The honey bee, itself cannot degrade the pectin, and therefore $G$. apicola might assist the honey bee in obtaining nutrients from the pollen. Snodgrasella alvi is another bacterium categorized as core gut member of honey bees. However, this bacterium was absent in the unexposed group. It was recognized only in the exposed group $[18,25]$. This result was somewhat contradictory to the previous studies, since $S$. alvi was also abundant in honey bee guts, while working together with G. apicola for acquisition of nutrients and for providing defense against pathogens by biofilm formation on the epithelium of the gut $[25,26]$. A 


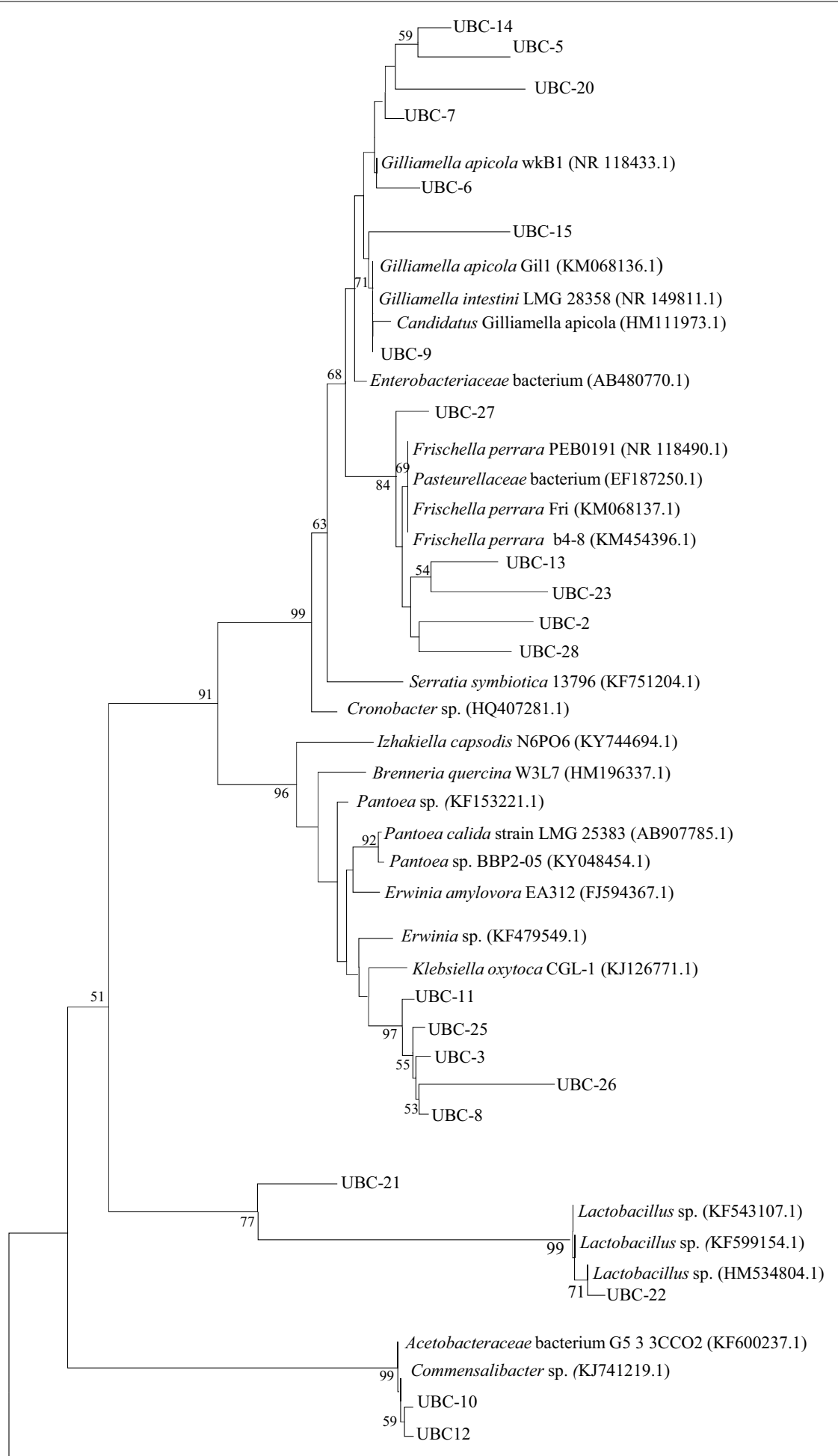

Helicobacter pylori 5443 (AH001595.2)

\subsection{0}

Fig. 2 Phylogenetic tree of the gut microbial clones of the unexposed bees. The evolutionary history was inferred using the neighbor-joining method and the evolutionary distances were computed using the Kimura-2 parameter. The percentage of replicate trees in which the associated taxa clustered together in the bootstrap test (1000 replicates) is shown next to the branches 


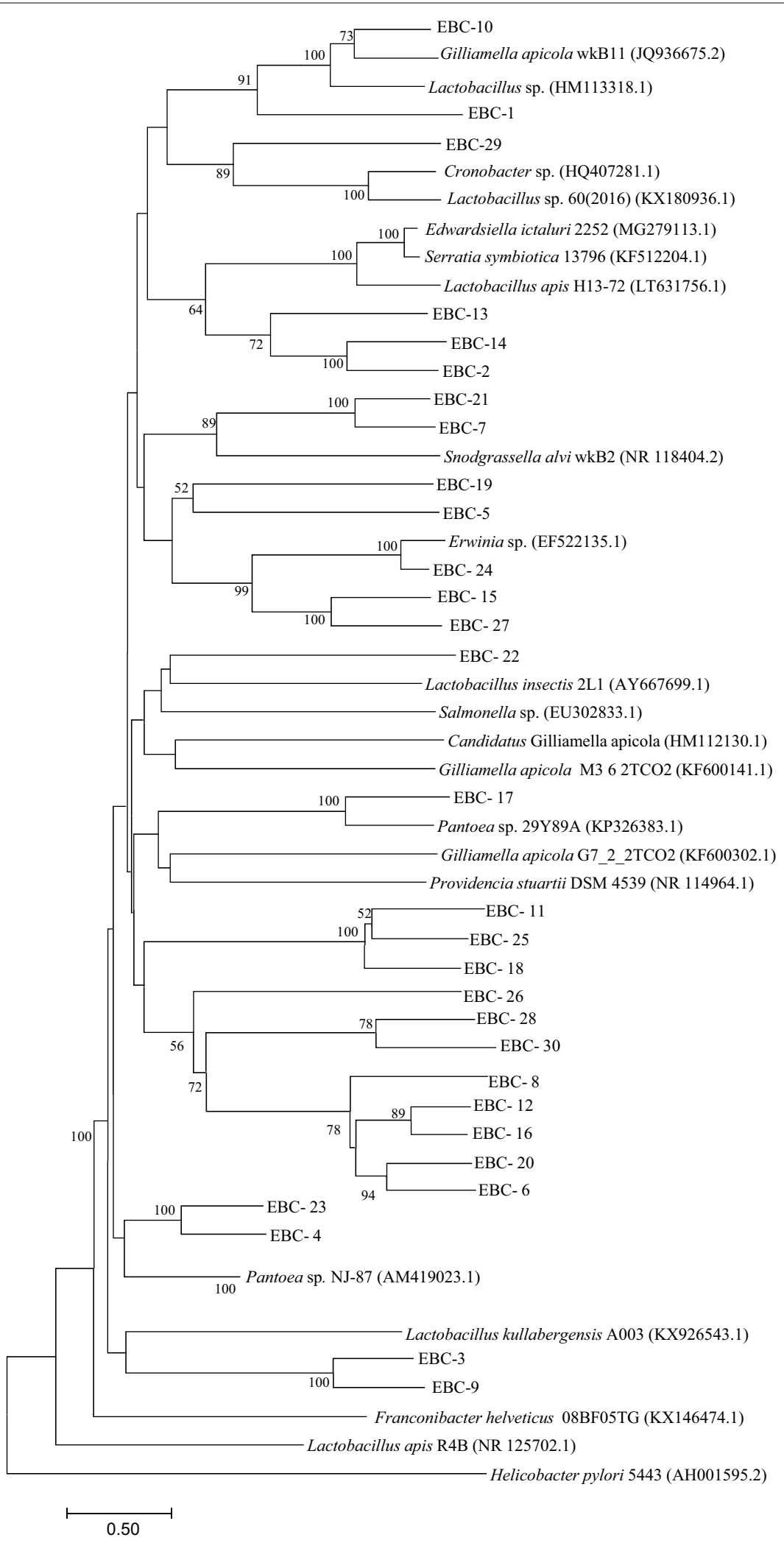

Fig. 3 Phylogenetic tree of the gut microbial clones of the exposed bees. The evolutionary history was inferred using the neighbor-joining method and the evolutionary distances were computed using the Kimura-2 parameter. The percentage of replicate trees in which the associated taxa clustered together in the bootstrap test (1000 replicates) is shown next to the branches 


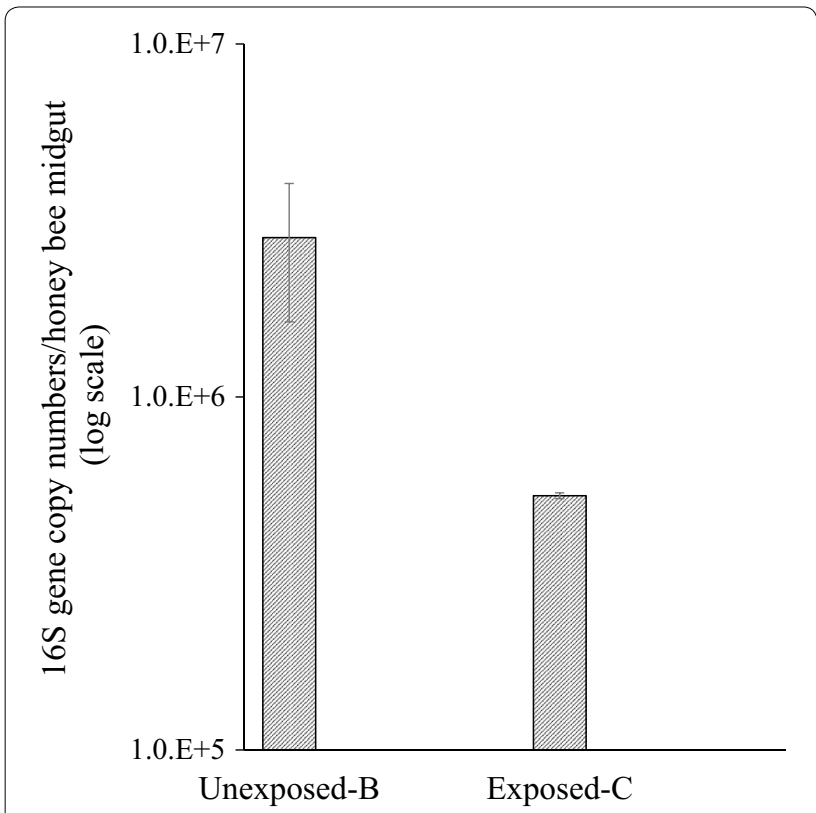

Fig. 4 Average copy numbers of 165 rRNA gene per gut of the unexposed and exposed honey bees to the pesticide of carbaryl. Single-factor ANOVA at 95\% confidence interval

prudent explanation to the absence of $S$. alvi in the unexposed bees might be that proportion of this core phylotypes occurred differently among individual bees, even if was is of the same age and from the same colony [3].

As enumerated by Bonilla-Rosso and Engel [24], the non-core bacterial groups included Frischella perrara, Bartonella apis, Lactobacillus kunkeei, Bombella apis, and Commensalibacter sp. Among these five groups of bacteria, only two were observed in this study. These were Commensalibacter and Frischella perrara, observed only in the unexposed bees. The Commensalibacter is an acetic acid bacterium (AAB), known as a commensal bacterium residing in the guts of insects whose diet is mainly derived from sugar-rich compounds like honey [27]. In relation to the result obtained in this study, the absence of this $\mathrm{AAB}$ in the exposed bees can possibly be suggestive of the health status of the exposed bees as explained by Crotti et al. [27]. On the other hand, F. perrara has been suggested to be responsible in scab formation in the ileum of the honey bee gut [25]. The scab formation is suggestive of immune regulation of the honey bees against the proliferation of $F$. perrara in the gut, although no study has yet reported any negative effect of $F$. perrara on honey bees.

Lastly, there were other bacteria which could be classified neither as core nor non-core bacteria. These bacteria included Cronobacter, Erwinia, Edwardsiella, Klebsiella, Providencia, Serratia, and Pantoea. The bacteria that are not commonly found in the gut microbiota of honey bees could have been acquired from the environment and could be considered as opportunistic pathogens [3, $25,28]$. These uncategorized bacteria were observed in more abundance in the exposed group as compared to the unexposed group. Klebsiella was only observed in the unexposed group, while Cronobacter, Edwardsiella, Providencia, Serratia, Erwinia, and Pantoea were observed in the exposed group. Encountering higher frequency of sequences similar to uncategorized bacteria could probably be indicative of disruption of balance of gut microbiome or disease as mentioned in previous studies in relation to dysbiosis in the presence of a potential cause like chemicals $[3,13,29]$. Although it is difficult to conclude decisively that the differences in the composition of the gut microbial communities from the two groups can be attributed directly to the pesticide exposure, Raymann et al. [13] have suggested that one difference between a healthy colony and a colony suffering from colony collapse disorder (CCD) can be a decrease in Alphaproteobacteria in gut bacterial communities [19]. This health condition and AAB proliferation is also attested by Crotti et al. [27]. This is in congruence with what was observed in the exposed bees, where there was absence of Alphaproteobacteria. Additional basis to infer variation between the two groups was comparison of the total copy number of bacterial 16S rRNA gene. The result showed that average gene copy number per bee gut was roughly an order higher in the unexposed bees with $2.83 \times 10^{6} \pm 1.20 \times 10^{6} \mathrm{copy} /$ bee than in the exposed bees with $5.25 \times 10^{5} \pm 9.81 \times 10^{3}$ copy/ bee, which was statistically significant at $95 \%$ confidence interval. Difference in the $16 \mathrm{~S}$ rRNA gene copy numbers seems to demonstrate effect of chemical exposure of the bees on the size of the gut bacterial communities. This result is well supported by the previous study indicating that the total gut bacterial abundance decreased among the bees exposed to tetracycline [13].

The results of this study showed the potential effect of carbaryl to the health of individual honey bees as indicated by the changes in composition of the microbial gut. Cumulative consequence of exposure to pesticides affecting the colony development of bumble bees [30], which is a close relative of honey bees, was clearly related to this. On the other hand, this study laid out a new aspect to look into the effect of pesticide in relation to the health of the individual worker bees which could, in cumulative effect, also affect the colony as worker bees carry out different tasks important to the maintenance of the colony $[17,19]$. However, the cumulative effect should be studied further to see if it is transitory or has little or no effect to the whole colony.

In the present study, we investigated the effect of transient exposure of honey bees to a pesticide, carbaryl. 
Based on the results obtained, there were differences in the members of the gut microbial communities between the unexposed and the exposed bees. These differences were observed in the composition of the core and noncore bacterial communities, as well as those bacteria not categorized as core or non-core gut members such as putative opportunistic pathogens. Moreover, the differences in the gut microbial communities became more apparent, when 16S rRNA gene copy numbers were compared between those groups. It would be valuable to determine in the future whether or not the effect of exposure to pesticides on the composition of the bacterial community is transitory, and therefore whether the bacterial community can be recovered.

\section{Authors' contributions}

$\mathrm{KN}$ carried out the molecular experiments and drafted the manuscript. SL participated in the molecular experiments and sequencing analysis. KC planned the study, performed the bee-pesticide experiments in the field chambers, and revised the manuscript. J-HL planned the study and revised the manuscript. All authors read and approved the final manuscript.

\section{Acknowledgements}

This research was supported by a Grant from the Research Program for Agricultural Science and Technology Development (Project No. PJ01351602), National Institute of Agricultural Science, Rural Development Administration, Republic of Korea, and in part by "Basic Science Research Program" through the National Research Foundation of Korea (NRF) funded by the Ministry of Education (NRF-2016R1D1A3B01012231).

\section{Competing interests}

The authors declare that they have no competing interests.

\section{Publisher's Note}

Springer Nature remains neutral with regard to jurisdictional claims in published maps and institutional affiliations.

Received: 5 December 2018 Accepted: 21 January 2019

Published online: 26 February 2019

\section{References}

1. Potts SG, Biesmeijer JC, Kremen C, Neumann P, Schweiger O, Kunin WE (2010) Global pollinator declines: trends, impacts and drivers. Trends Ecol Evol 25:345-353

2. Johnson RM, Ellis MD, Mullin CA, Frazier M (2010) Pesticides and honey bee toxicity_USA. Apidologie 41:312-331

3. Kwong WK, Moran NA (2016) Gut microbial communities of social bees. Nat Rev Microbiol 14:374

4. Chon K, Lee H, Hwang HC, Im J, Park K-H, Paik MK, Choi Y-S (2017) The honey bee brood test under semi-field conditions for the assessment of positive reference chemicals in Korea. Appl Biol Chem 60:569-582

5. Aizen MA, Garibaldi LA, Cunningham SA, Klein AM (2009) How much does agriculture depend on pollinators? Lessons from long-term trends in crop production. Ann Bot 103:1579-1588

6. Kluser S, Peduzzi P (2007) Global pollinator decline: a literature review. UNEP/GRID, Geneva

7. Sanchez-Bayo F, Goka K (2014) Pesticide residues and bees-a risk assessment. PLoS ONE 9:e94482
8. Moonsu K, Chuleui J (2017) Avoidance behavior of honey bee, Apis mellifera from commonly used fungicides, acaricides and insecticides in apple orchards. J Apic 32:295-302

9. MacKenzie KE, Winston ML (1989) The effects of sublethal exposure to diazinon, carbaryl and resmethrin on longevity and foraging in Apis mellifera L. Apidologie 20:29-40

10. Biddinger DJ et al (2013) Comparative toxicities and synergism of apple orchard pesticides to Apis mellifera (L.) and Osmia cornifrons (Radoszkowski). PLOS ONE 8:e72587

11. Berenbaum MR (2016) Does the honey bee "risk cup" runneth over? Estimating aggregate exposures for assessing pesticide risks to honey bees in agroecosystems. J Agric Food Chem 64:13-20

12. Corby-Harris $V$, Maes $P$, Anderson KE (2014) The bacterial communities associated with honey bee (Apis mellifera) foragers. PLoS ONE 9:e95056

13. Raymann K, Shaffer Z, Moran NA (2017) Antibiotic exposure perturbs the gut microbiota and elevates mortality in honeybees. PLoS Biol 15:e2001861

14. Vojvodic S, Rehan SM, Anderson KE (2013) Microbial gut diversity of Africanized and European honey bee larval instars. PLoS ONE 8:e72106

15. Lim E-S (2018) Preparation and functional properties of probiotic and oat-based synbiotic yogurts fermented with lactic acid bacteria. Appl Biol Chem 61:25-37

16. Chen X, Song J-L, Hu Q, Wang H, Zhao X, Suo H (2018) Positive enhancement of Lactobacillus fermentum HY01 on intestinal movements of mice having constipation. Appl Biol Chem 61:39-48

17. Engel P, Martinson VG, Moran NA (2012) Functional diversity within the simple gut microbiota of the honey bee. Proc Natl Acad Sci USA 109:11002-11007

18. Engel P, Moran NA (2013) The gut microbiota of insects-diversity in structure and function. FEMS Microbiol Rev 37:699-735

19. Schwarz RS, Moran NA, Evans JD (2016) Early gut colonizers shape parasite susceptibility and microbiota composition in honey bee workers. Proc Natl Acad Sci USA 113:9345-9350

20. Engel P, James RR, Koga R, Kwong WK, McFrederick QS, Moran NA (2013) Standard methods for research on Apis mellifera gut symbionts. J Apic Res $52: 1-24$

21. Ritalahti KM, Amos BK, Sung Y, Wu Q, Koenigsberg SS, Löffler FE (2006) Quantitative PCR targeting 16S rRNA and reductive dehalogenase genes simultaneously monitors multiple Dehalococcoides strains. Appl Environ Microbiol 72:2765-2774

22. Engel P, Moran NA (2013) Functional and evolutionary insights into the simple yet specific gut microbiota of the honey bee from metagenomic analysis. Gut Microbes 4:60-65

23. Moran NA (2015) Genomics of the honey bee microbiome. Curr Opin Insect Sci 10:22-28

24. Bonilla-Rosso G, Engel P (2018) Functional roles and metabolic niches in the honey bee gut microbiota. Curr Opin Microbiol 43:69-76

25. Engel P, Bartlett KD, Moran NA (2015) The bacterium Frischella perrara causes scab formation in the gut of its honeybee host. mBio 6:e00193-00115

26. Kwong WK, Engel P, Koch H, Moran NA (2014) Genomics and host specialization of honey bee and bumble bee gut symbionts. Proc Natl Acad Sci USA 111:11509-11514

27. Crotti E et al (2010) Acetic acid bacteria, newly emerging symbionts of insects. Appl Environ Microbiol 76:6963-6970

28. Raymann K, Moran NA (2018) The role of the gut microbiome in health and disease of adult honey bee workers. Curr Opin Insect Sci 26:97-104

29. Ahn J-H, Hong I-P, Bok J-I, Kim B-Y, Song J, Weon H-Y (2012) Pyrosequencing analysis of the bacterial communities in the guts of honey bees Apis cerana and Apis mellifera in Korea. J Microbiol 50:735-745

30. Gill RJ, Ramos-Rodriguez O, Raine NE (2012) Combined pesticide exposure severely affects individual- and colony-level traits in bees. Nature 491:105 